\title{
Sistema de Apoio ao Gerenciamento de Configuração dos Laboratórios de Informática do IFSul Campus Charqueadas
}

\section{RESUMO}

As organizações estão usando cada vez mais a tecnologia diante da competitividade nos mercados em que atuam. Nesse contexto, a indisponibilidade dos serviços ofertados afeta a imagem da organização, além de prejuízos financeiros. A partir de interações realizadas com o setor de Tecnologia da Informação (TI) do IFSul Câmpus Charqueadas, constatou-se que este não possui uma ferramenta automatizada para auxiliar na gestão dos laboratórios de informática, tornando as rotinas de manutenção preventiva e corretiva propensas a falhas humanas, por serem tratadas manualmente. Sendo assim, o objetivo deste projeto e desenvolver um sistema Web de apoio a Gestão de TI para os laboratórios de Informática da instituição, que atualmente são 12 laboratórios, totalizando 220 computadores, que atendem aproximadamente 1000 alunos de nível médio, técnico, superior, pós-graduação e mestrado. A metodologia para este trabalho envolveu atividades como o estudo dos padrões e melhores práticas relacionados à gestão de TI, tendo como base teórica o capítulo de Gerenciamento de Configuração do Information Technology Infrastructure Library (ITIL), além de reuniões para levantamento de requisitos junto a equipe de TI para investigação de ferramentas de suporte ao desenvolvimento da solução proposta, implementação de uma prova de conceito, e validação dos testes. A solução proposta dispõe de dois módulos principais: (i) o modulo automatizado que utiliza à ferramenta OpenSource intitulada como Open Computer and Software Inventory Next Generation (OCS Inventory), responsável por fazer a coleta e armazenamento das informações dos computadores; (ii) e o módulo Web capaz de ler a base de dados gerada pelo OCS, oferecendo um conjunto de funcionalidades que auxiliam a equipe de TI na visualização de informações e no suporte a tomada de decisões, como relatórios de hardware, software, notificações e representação gráfica dos laboratórios.

Palavras-chave: Gerenciamento dos Serviços de Tecnologia da Informação, ITIL, Gerenciamento de Configuração, OCS Inventory, Laboratórios de Informática.

\section{INTRODUÇÃO}

Atualmente, as organizações são dependentes dos recursos e serviços fornecidos pela Tecnologia da Informação e Comunicação (TIC). Neste sentido, são obrigadas a fazer uso da tecnologia de uma forma mais intensa para obter mais competitividade e qualidade em seu mercado de atuação [1]. Os setores responsáveis pela gestão tecnológica, tem a missão primordial de manter a infraestrutura e os serviços providos em funcionamento, preferencialmente no modelo $24 \times 7$ que é um modelo onde os profissionais de suporte prestam serviços para os usuários finais da área de TI no formato vinte e

\footnotetext{
${ }^{1}$ IFSUL - Instituto Federal de Educação Ciência e Tecnologia Sul-Rio-grandense - Câmpus Charqueadas - Brasil.

2 IFSUL - Instituto Federal de Educação Ciência e Tecnologia Sul-Rio-grandense - Câmpus Charqueadas - Brasil.
} 
quatro horas por dia, durante sete dias da semana, atuando no suporte aos usuários e prevenção de incidentes [2]. Caso contrário, degradações nos serviços ofertados podem culminar, em perdas financeiras, além da degradação da imagem da corporação/organização. Entretanto, introduzir TIC no suporte a serviços requer planejamento e análise dos custos envolvidos comparados com as vantagens mercadológicas almejadas [1].

Diante desse cenário, dada a complexidade e evolução contínua requerida para uma gestão adequada de Tecnologia da Informação (TI), surgem padrões e disciplinas que direcionam os fluxos e processos na área. Especificamente, tem-se o Gerenciamento de Serviços de Tecnologia da Informação (GSTI) que se consolida como uma ferramenta de gestão importante, instruída com uma visão de unificar pessoas, processos e tecnologia, buscando de fato desenhar soluções alinhadas com os objetivos estratégicos da empresa. Para concretizar seus objetivos, o GSTI disponibiliza diversos frameworks, dentre eles tem-se o ITIL, que se consolida por reunir uma série de procedimentos, tendo como destaque o Gerenciamento de Configuração, que tem como objetivo, controlar os meios de produção que são pontos cardeais para a criação de produtos ou serviços a serem oferecidos, esses meios de produção são conhecidos neste processo como Itens de Configuração (IC) que são componentes no qual fazem parte ou estão diretamente relacionados com a infraestrutura de TI, por exemplo: Computadores e Softwares instalados.

Mediante o que foi exposto, por consequência de o setor de TI do IFSul gerenciar manualmente seus ativos estabelecidos nos laboratórios de informática, sem o uso de uma ferramenta computacional. Este trabalho consisti em utilizar como base as boas práticas indicadas pelo gerenciamento de configuração do ITIL, com o foco em implantar um modulo integrado a ferramenta open source OCS Inventory, concebendo ao fim um sistema para controle de inventario da infraestrutura de hardware e software dos laboratórios de informática, principalmente em conduzir a adição de técnicas de visualização de informações que venham a auxiliar no processo de tomadas de decisões táticas e estratégicas da equipe de TI.

\section{FUNDAMENTAÇÃO TEÓRICA}

Esta seção tem como objetivo, apresentar uma visão geral sobre os principais conceitos que o GSTI tem a dispor para o presente trabalho, principalmente na aplicação das melhores práticas do framework ITIL, parte interessada no que diz respeito ao processo de gerenciamento de configuração juntamente com a ferramenta computacional Open-Source OCS Inventory, na sua agregação diante do contexto da infraestrutura de ensino do IFSul Campus Charqueadas.

\subsection{Gerenciamento dos Serviços de Tecnologia da Informação}

O GSTI como um todo é composto por pessoas, processos e tecnologias, juntos cooperam na garantia da qualidade dos serviços, com apoio a diversos níveis conforme as necessidades da organização [3]. Para melhor assimilação, ao agrupar as atividades dentro de um processo, torna-se mais fácil de controlar o ambiente, possibilitando gerar resultados e acompanhamento do seu desempenho. Além disso, tecnologia é um fator indispensável, uma vez que a mesma concebe maior automatização das atividades em paralelo com as pessoas que são as partes interessadas neste contexto [3]. De acordo com essa perspectiva, segundo o autor [3] para adotar o GSTI " é preciso levar em conta que a maioria dos benefícios de um programa de Gerenciamento de Serviços podem levar um tempo para serem obtidos, dispondo também de proveitos a curto prazo para a empresa que implementar esta 
metodologia, gerando maior agilidade, satisfação dos clientes, qualidade no serviço, redução de custos, segurança e confiabilidade, fornecimento de informações para avaliar desempenhos, e equipes de TI mais motivadas." Com base no que foi exposto, assim que o GSTI e inserido em uma organização o autor [4] revela que o mesmo disponibiliza frameworks a serem aplicados sob diversos aspectos, seja na Governança de TI muito bem reforçado pelo Control Objectives for Information and Related Technologies (COBIT), na qualidade da gestão pela ISO 20000 ou operacionalmente no ITIL, que se diferencia dos demais mencionados, por conter o gerenciamento de configuração, processo principal para organização das demandas de suporte a serviços, na qual o existente trabalho se comporta.

\subsection{ITIL}

Framework selecionado, pois aborda melhorias em processos de gerenciamento de configuração, com propósito de otimização e identificação de meios críticos. Além disso, possui amplas recomendações de diversas empresas públicas e privadas no mundo, sendo atualmente um padrão no quesito de boas práticas para GSTI. De acordo com Foina [5] o ITIL "foi inicialmente publicada em 1989 pela Central Computer Telecommunication Agency (CCTA) atualmente conhecida por Office of Government Commerce (OGC), Agencia do Reino Unido. Desde então, se propõe a ser uma fonte de boas práticas de GSTI, baseada na experiencia de diversas empresas no mundo e organizadas por um grupo de renomados especialistas em computação e administração". Com o passar dos anos foi se destacando vigorosamente, sendo hoje a abordagem mais utilizada no mundo entre as metodologias aplicadas na governança de TI, conforme mostra a pesquisa realizada pela International Network Services onde 194 organizações foram consultadas e 39\% responderam que utilizam este framework [6]. Outrossim, uma pesquisa realizada pela Information Technology Service Management (ITSMF), informa que 33\% das empresas consultadas utilizam o ITIL. Em seguida, aparece o COBIT com 16\%, o Balanced Scorecard com 11\% e o CMMI e PMI com 6\% [7]. Segundo o autor [3], podese destacar como os principais benefícios: (i) Melhoraria da qualidade dos serviços garantindo consistência na entrega, atendendo as necessidades de negócio, (ii) Redução dos custos operacionais, (iii) investimentos em TI, (iv) e processos mais eficientes.

Muitas dessas melhores práticas citadas, são exemplificadas por meio dos sete livros que o ITIL possui em sua atual versão, descrevendo como os processos podem ser formalizados. Segundo [3] dentre os sete livros, tem-se o de "Suporte a Serviços" que especifica o "Gerenciamento de Configuração", o qual será abordado na próxima seção, pois é o tema no qual está centrado o presente trabalho.

\subsection{Gerenciamento de Configuração}

Tem como objetivo fornecer um modelo logico da infraestrutura de TI, possibilitando o gerenciamento pleno dos ativos, denominados como IC. Segundo [6] essencialmente, o ITIL não restringe o conceito de IC mas, via de regra, este e categorizado como um componente que faz parte ou está diretamente relacionado na infraestrutura, tais como: Computadores (Hardware), aplicativo ou sistema (software), manual técnico do equipamento ou procedimento de trabalho. Entretanto, estes IC, produzem muitas informações, e as mesmas, de certa forma precisam estar em algum local acondicionado. Nesse sentido, tem-se o Banco de Dados de Gerenciamento de Configurações (BDGC) responsável pelo armazenamento das informações [8]. Estes aspectos mencionados, são fundamentais para os laboratórios de informática do IFSul, possibilitando maior controle no inventario de Hardware e Software, com o objetivo de manter o bom funcionamento dos IC contribuindo nas tomadas de 
decisões. Segundo o autor [6] as principais atividades do processo de Gerenciamento de Configuração, são as seguintes: (i) Visão mais ampla da infraestrutura, auxiliando no planejamento de TI; (ii) identificação dos IC; (iii) controle de entrada e saída dos IC; (iv) registro do estado; (v) verificação e auditoria, e (vi) geração de informações para auxílio as tomadas de decisão.

Diante desse segmento, Castilho et al. [9] afirma que existem diversas ferramentas para apoio ao gerenciamento de configuração. A seguinte Subseção II-D aborda os tipos de ferramentas.

\subsection{Ferramentas para Gerenciamento de Configuração}

Com a progressão da TI, atualmente o fluxo de informações vem cada vez mais se expandindo dentro das organizações, e para ter o controle das mesmas temos diversas ferramentas para controle dos IC, Ferreira [10] afirma que essas ferramentas, "efetuam uma função crucial garantindo ao setor de TI uma análise minuciosa dos seus ativos acarretando decisivamente em uma melhoria contínua no desempenho dos IC". Essa colocação do autor, vem ao encontro dos equipamentos operando regularmente, pois quando o desempenho é mensurado de forma continua a infraestrutura de TI só tem a ganhar com organização a prevenção de possíveis falhas.

Nesse contexto, Castilho et al. [9] diz que as ferramentas para gerenciamento de configuração tem como intenção, "tornar o processo de gerencia de ativos, inventário das redes envolvidas, controle de insumos e suporte técnico mais eficiente."' Perante a isto, sabendo da importância de monitorar os IC, existem diversas ferramentas proprietárias e open source. De acordo com as bases teóricas apresentadas em [11] e [12], bem como no trabalho apresentado por Mello e Santos [13], tem-se por exemplo diversas ferramentas computacionais para apoio ao gerenciamento de configuração, tais como: PRTG Network Monitor, Nagios, Cacic e a ferramenta OCS Inventory, escolhida para este trabalho, em virtude de uma fácil implementação, e uma comunidade ativa incluindo grupos empresarias com pessoas de diversos países, sendo uma ferramenta Open Source, multiplataforma e principalmente por dispor dos requisitos que atendem as necessidades do setor de TI do IFSul [14].

\subsection{OCS Inventory NG}

Em vista dos argumentos apresentados nas seções anteriores o gerenciamento de configuração requer o uso de ferramentas para permitir o controle do parque tecnológico. Conforme dito, a ferramenta selecionada para este trabalho foi o OCS Inventory, que permite aos gestores de TI, realizar a coletar das informações de Hardware e Software dos ativos localizados na rede, através da execução do programa cliente ("OCS Inventory Agent") instalado na máquina, permitindo de forma automática o envio das informações para o banco de dados relacional MySQL, que se encontra em uma estação Gerente/Servidor OCS configurada para realização da interação entre Agentes e o Gerente. Segundo Alves [15] com ele é possível registrar todas as características de um computador incluindo: Versão do sistema operacional, número de série do equipamento, marca, modelo, características dos componentes de hardware e softwares instalados, dentre outros dados. Scota [16] define que o OCS como um software de inventario, "muito importante para o gerenciamento de uma rede, já que este permite mapear todo o parque de máquinas, suas configurações de hardware e informações de software. Com estes dados em mãos é possível responder, rapidamente, dúvidas comuns como sobre a performance de computadores, informações de acesso remoto, sistemas e aplicativos sem licença ou desatualizados, dentre outros aspectos. Além disso, com um inventário atualizado, e possível identificar o número exato de computadores e dispositivos em uso, em estoque, 
em manutenção e que estão dentro ou fora da garantia, evitando erros em tomadas de decisões estratégicas, como em novos investimentos."

\subsection{Principais Características do OCS}

Para Mattar [17] o OCS Inventory tem como característica principal uma arquitetura de comunicação disponível em três plataformas distintas: Windows, MAC e Linux. Além disso, possui uma arquitetura de comunicação cliente-servidor "onde os clientes correspondem a agentes que são instalados nas máquinas que se pretendem controlar." [10]. Com a utilização dos protocolos HTTPS e SNMP, o OCS e constituído de quatro componentes principais:

- Servidor de banco de dados: Responsável por armazenar informações de inventário, em seu BDGC que é o MySQL.

- Servidor de comunicação: Manipula as comunicações HTTP e SNMP entre o servidor de banco de dados e agentes.

- Console de administração: que permite aos administradores consultar o servidor de banco de dados usando o navegador favorito.

- Servidor de implantação: que armazena todas as configurações de implantação do pacote.

Em vista dos argumentos apresentados, esses quatro principais componentes podem ser hospedados em um único computador ou em computadores diferentes permitindo balancear sua carga.

\subsection{Requisitos para Configuração}

Máquina virtual ou Servidor com sistema operacional Ubuntu Server 16.10, com os principais requisitos para implantação da estação Gerente do OCS de acordo com o repositório GitHub da própria ferramenta [18], ou pelo site [19], que são eles: (i) Instalação dos pacotes básicos, (ii) Instalação e configuração do serviço Web Apache com suporte a linguagem de programação PHP, (iii) Instalação do PHP com a extensão php-mysql, e (iv) Instalação e configuração do banco de dados MySQL.

\section{TRABALHOS RELACIONADOS}

Nesta Seção, serão apresentados os trabalhos relacionados, onde inicialmente tem-se, na Subseção 3.1, intitulado como "Gerenciamento dos Serviços de TI em instituição de Ensino Superior" [20]. Na Subseção 3.2, tem-se o trabalho nomeado como "GAIA Inventario: Um Modelo para Gestão da Configuração, Inventário e Ativos de Serviços de Tecnologia da Informação" [21]. Por fim, na Subseção 3.3, é exibido um comparativo entre as soluções em perspectiva com o trabalho proposto.

\subsection{Gerenciamento dos Serviços de TI em instituição de Ensino Superior}

Na solução 3.1 [20], o principal foco está na aplicação das melhores práticas sugeridas pela ISO/IEC 20000 que explica o gerenciamento de qualidade do GSTI, juntamente com o COBIT para a Governança de TI e o ITIL para o gerenciamento de configuração, para que juntas possam atuar no aprimoramento da gestão dos serviços de TI da Associação educacional Dom Bosco (AEDB). A AEDB se caracteriza por ter um ambiente heterogêneo de demandas, atendendo diferentes níveis de ensino, desde fundamental a Cursos de Pós-graduação e Extensão (CPGE), cursos em convênio com a Fundação Getúlio Vargas (FGV) e o curso de mestrado em Engenharia de Produção em convênio com a UNESP Guaratinguetá, estimando 2850 clientes ao todo, fracionados, entre 2500 alunos e 200 
professores que são o seu público alvo. Isso aproxima o trabalho da presente proposta, uma vez que o IFSul Câmpus Charqueadas também abrange o ensino verticalizado, com um público de ensino entre ensino médio e mestrado, com 902 alunos em média, empregando o uso de 220 computadores distribuídos em 12 laboratórios até a data atual do presente trabalho.

Diante do contexto apresentado pelo autor, o principal problema e a não existência de metodologias para controle dos ativos, registro de incidentes e monitoramento de dispositivos e sistemas. Com isso, a gestão de TI acontecia de forma, tipicamente manual. Essa forma de trabalho acabava por gerar impactos diretos nas atividades da instituição, refletindo prejudicialmente nos recursos didáticos, bem como na qualidade de ensino.

Diante do problema exposto, o autor baseou-se no conjunto de boas práticas já mencionadas anteriormente, especificamente ao gerenciamento de configuração tratando mais especificamente na abordagem e definição dos tipos de IC, a fim de garantir um controle eficaz dos mesmos incluindo informações como situação dos ativos, versão, localização, relacionamento com outros IC e problemas associados. A partir disso o autor classificou as ferramentas que melhor se adaptam dentro do contexto da AEDB. Sendo assim, o Gerenciamento de serviços foi habituado em ferramentas Open Source, a fim de otimizar o processo dos ativos, com uma solução composta por: (i) GLPI [22]; (ii) Zabbix [23]; e (iii) OCS Inventory que também é utilizado no presente trabalho.

Assim sendo, III-A tem como diferencial a adaptação do sistema de eventos que resultam em uma ação dentro do GLPI, de tal forma que o Zabbix, ao detectar alguma irregularidade na rede, aciona um Web Service que e responsável por abrir um chamado no GLPI, automaticamente. Dessa forma, o evento e registrado com a descrição do componente no qual está necessitando de um reparo. 0 OCS Inventory também executa uma ação integrada ao GLPI. Onde toda vez que um computador é iniciado, o agente que está instalado nesta máquina coleta as informações e atualiza, se necessário, no BDGC que é sincronizado através de um plugin do GLPI. Após o fim desta sincronização todas as informações ficam disponíveis para consulta e associação de chamados. Outra funcionalidade é via e-mail, onde os usuários dos serviços de TI podem criar chamados através do e-mail atendimento@aedb.br, permitindo maior flexibilidade para tomada de decisões gerando ao fim este resultado.

\subsection{Gaia Inventário: Um Modelo Para Gestão da Configuração, Inventário e Ativos de Serviços de Tecnologia da Informação}

Na solução apresentada por Honda [21], o modelo e baseado nas boas práticas do ITIL para Gerenciamento de Configuração, e no COBIT para governança de TI, com objetivo de garantir eficiência em seu ambiente, auxiliando os gestores nas atividades recorrentes no dia a dia, favorecendo o ambiente no controle e otimização diante das tomadas de decisões relacionadas ao registro de estado, auditoria, relatórios e verificação do próprio Laboratório Gaia, onde foi implementado. Isso aproxima o trabalho com a presente proposta, uma vez que os laboratórios de informática do IFSul Campus Charqueadas também são constituídos por IC, utilizados para fins didáticos.

Diante do que foi mencionado, o principal problema é a falta de informações dos IC para controle e histórico de informações tais como: hardware, rede, sistema operacional e identificação física. Para tal, foi utilizada uma abordagem bottom-up que envolve inventariar todos os equipamentos aplicados 
à infraestrutura da organização, atrelado ao BDGC que consiste em cadastrar os IC e suas informações no banco de dados. Sendo assim, a sua arquitetura é dividida da seguinte forma: (i) modulo de descoberta de máquinas composto por agentes multiplataforma desenvolvido com o uso da linguagem Python, configurados com a Uniform Resource Locator (URL) do servidor, gerando um código identificador do local onde a máquina se encontra, com a coleta acontecendo através de serviços RESTful implementados em um servidor Representational State Transfer (REST) com o uso da linguagem Java e o Framework Jersey essencial para a implementação de um Web Service; e (ii) módulo Web, Desenvolvido com a linguagem PHP e o framework Yii para comunicação Server-Side, especificamente ao BDGC, apresentando as informações dos IC na tela, com as funcionalidades: (a) Gerenciamento de Locais, com o uso da API V3 do Google Maps para exibir detalhes de geolocalização dos locais cadastrados; (b) Gerenciamento de Máquinas, capaz de gerar relatórios sobre garantia, sistema operacional e informações de hardware; e (c) Gerenciamento de Equipamentos que permite consulta dos IC, com informações do seu tipo, onde ambos módulos tem relação com um BDGC que recebe as informações dos IC via REST através do Módulo Descoberta de Máquinas, e armazena em seu banco de dados que PostgreSQL.

Em suma, o modelo Gaia Inventário e capaz de gerar relatórios com detalhamento de diversa informações de Hardware dos IC, bem como relatório de garantia. Estes relatórios gerados, facilitam que as auditorias dos IC sejam feitas de forma rápida e eficaz, com capacidade também de gerar gráficos das informações.

\subsection{Comparativo}

Diante do que foi demonstrado nos dois trabalhos relacionados, percebe-se que ambos têm o foco no gerenciamento de configuração e armazenamento dos dados em um BDGC, baseando-se no ITIL. Onde em cada um dos trabalhos, os autores detêm objetivos semelhantes que são eles: Agentes para coleta, armazenamento em um BDGC e uma interface Web para exibir as informações dos IC. Nesse sentido, os mesmos são próximos ao presente trabalho, que se diferencia por utilizar as informações coletadas dos IC para auxílio nas manutenções preventivas, não somente por relatórios, mas também por um layout dos laboratórios representados via $W e b$, com base no atual status e distribuição física dos computadores.

\section{SOLUÇÃO PROPOSTA}

Na presente seção será apresentado inicialmente o ambiente do IFSul Câmpus Charqueadas e o levantamento de requisitos realizado (Seções 4.1 e 4.2). Posteriormente, é apresentada a visão geral da solução proposta e as funcionalidades desenvolvidas (Seção 4.3). Na Seção 4.4 são especificadas as tecnologias e ferramentas de apoio utilizadas durante o desenvolvimento do sistema. Por fim, são apresentados os resultados obtidos na Seção 4.5.

\subsection{OCS Inventory no ambiente do IFSul Câmpus Charqueadas}

Atualmente, o IFSul Campus Charqueadas possui uma infraestrutura com doze laboratórios de informática somando duzentos e vinte computadores. Estes laboratórios, são de fundamental importância para a qualidade do ensino, pois dão suporte aos sete cursos disponibilizados, da mesma forma para os projetos de pesquisa e extensão. Sob essa perspectiva, e com base no que foi dito nas seções anteriores, para administrar os laboratórios de informática, apresenta-se como solução 
proposta o desenvolvimento de um sistema baseado nas boas práticas do GSTI, com ênfase no suporte a serviços do ITIL, alinhado o processo de gerenciamento de configuração integrado à ferramenta OCS Inventory, colaborando de maneira ágil na tomada de decisões por parte do setor de TI, e de forma preventiva, impedindo falhas no desempenho dos computadores, bem como garantia da qualidade no ensino da instituição.

\subsection{Levantamento de requisitos}

Para que as funcionalidades de usuário fossem adequadas às necessidades da equipe de TI, fez-se necessário realizar entrevistas junto aos mesmos. As entrevistas foram realizadas através questionários com perguntas abertas referente ao método de gerenciamento de configuração do setor de TI. Os resultados obtidos nas entrevistas, apontaram as principais funcionalidades para o controle dos laboratórios, tais como: (i) inventario dos softwares que estão sendo instalados, (ii) inventário dos tipos de computadores e suas informações de hardware, (iii) acesso único para cada usuário, (iv) notificações caso ocorra alguma alteração, (v) layout dos laboratórios representando o seu atual contexto físico com o status atual de cada computador.

\subsection{Visão Geral e Funcionalidades}

A arquitetura do atual trabalho, foi desenvolvido por dois módulos que são eles: (i) O Módulo automatizado, e (ii) o Módulo Web, conforme ilustra a Figura 2 a seguir que apresenta a visão geral da solução proposta.

O Modulo automatizado, utiliza a estrutura do OCS Inventory que trabalha de forma automática, começando inicialmente na atuação dos agentes em Perl, coletando os dados dos computadores, logo após enviando em formato XML para a estação gerente/servidor do OCS, que dispõe de um BDGC que e o MySQL, onde fica armazenado toda a base de dados até então tudo isso acontece em tempo real da coleta, sendo ao inserir novos dados, ou atualizando o que já tem disponível no banco.

Figura 1. Arquitetura da visão geral de toda a aplicação

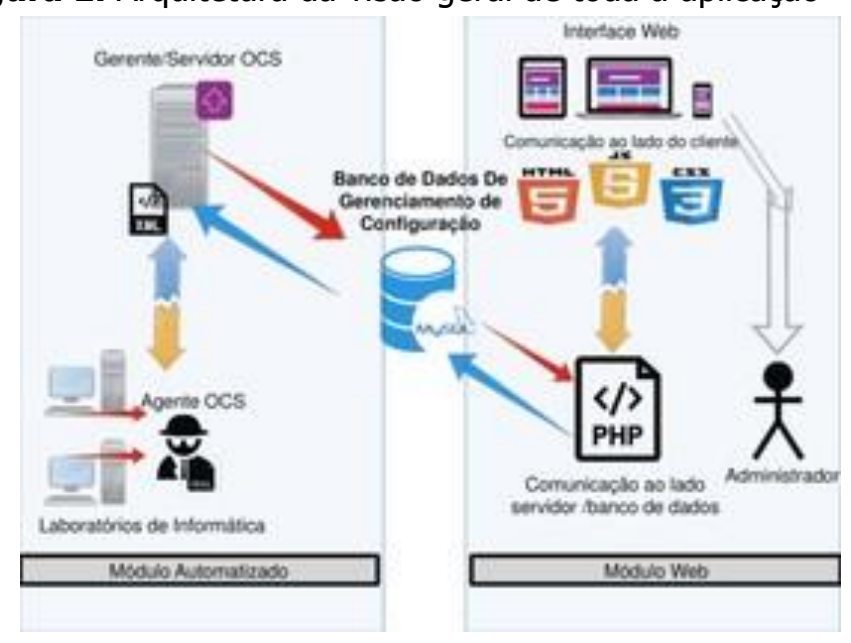

Com o OCS apresentando maturidade suficiente em todos os seus requisitos de configuração, mencionados na II-G, agindo de forma automática, desde a coleta até o armazenamento dos dados, tem-se então o Módulo Web, com foco na visão do usuário comunicando-se com os agentes do OCS e o BDGC, com todo seu desenvolvimento ao lado do cliente e do servidor, desenvolvido de modo autoral, com uma tela, distribuída em menus específicos, de acordo com o levantamento de requisitos abordado na Subseção 4.2 tais como: cadastro e login para controle de acesso a usuários, 
notificações, laboratórios disponíveis representados no sistema por cards contendo informações detalhadas de cada laboratório, onde os usuários responsáveis pela operação do sistema que são os profissionais de TI da instituição, podem escolher qual laboratório pretendem obter informações. Clicando em um desses cards, e possível ir para a tela de detalhas do laboratório selecionado, englobando os principais detalhes dos computadores inseridos, tais como: Layout dos laboratórios, faixa de rede, lista dos softwares, informações de hardware, marca, modelo, quantidade de computadores, data de fabricação e etc.

\subsection{Ferramentas e tecnologias utilizadas}

Além do módulo automatizado configurado em uma estação gerente/servidor Linux, contendo agentes em Perl, e BDGC em MySQL, o desenvolvimento back-end da aplicação foi desenvolvido com o uso da linguagem PHP juntamente com a biblioteca Smarty Template e o padrao Model-View-Controller (MVC) para organização dos scripts, afim de interagir com a interface front-end, que consiste em três tecnologias que são elas: HTML, CSS e JavaScript. Com essas três tecnologias combinadas, foi utilizado os seguintes frameworks, tais como: Bootstrap 4, Font Awesome, Ajax e JQuery. Outrossim, para agilidade e controle do desenvolvimento do trabalho, foi utilizado três ferramentas, que são elas: Git, GitHub e Trello. De forma mais específica o Git foi usado para versionamento de código e histórico de alterações, e como como meio de armazenamento do sistema em um repositório, foi utilizado o GitHub. Assim como ocorreu versionamento e armazenamento remoto dos códigos-fontes, as atividades do sistema foram agilizadas ao longo de seu desenvolvimento através do Trello que e um ferramenta de qualidade que funciona em um formato de Kanban, com o propósito de separar as atividades em etapas que são: (i) A fazer, (ii) em andamento e (iii) concluído.

\subsection{Resultados}

Com a base de dados provida de informações vindas pelo módulo automatizado do OCS, foi necessário entender todas as tabelas geradas, a partir disto relacionando cada uma delas e depois criando métodos responsáveis por fazer o módulo Web entender o que seria um laboratório, quais os seus computadores, softwares instalados, e demais informações estas e demais funcionalidades foram criadas inicialmente com a nomenclatura dos computadores na rede, por exemplo, os computadores do laboratórios dois, tem a sua nomenclatura como: PC-201, PC202, o laboratório sete, tem a sua nomenclatura como: PC701, PC-702 e assim sucessivamente. A Figura 2 a seguir mostra a tela inicial do sistema.

Figura 2. Demonstração da tela inicial do sistema após o login, com seus menus e cards que representam cada laboratório disponível para análise dos detalhes

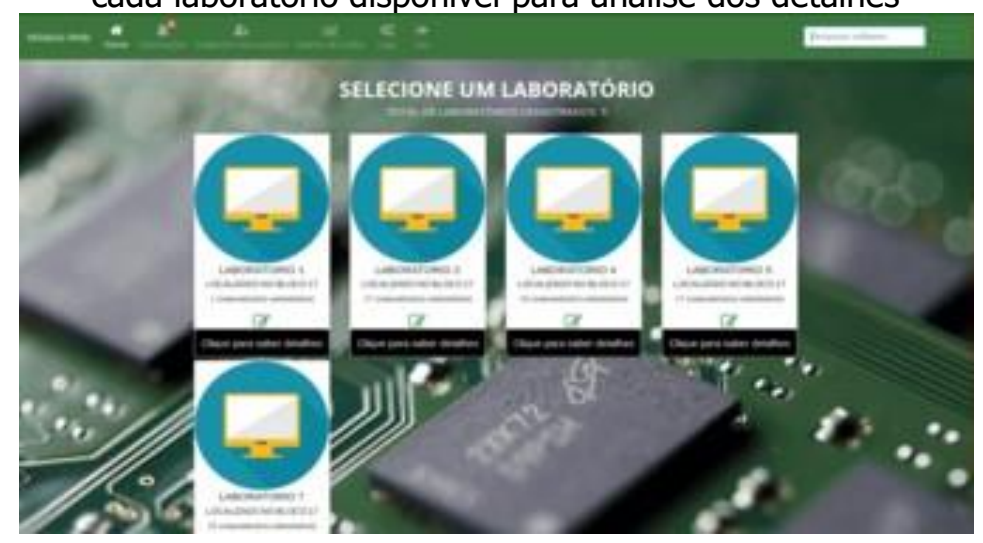




\section{CONCLUSÕES}

O presente trabalho detalha o desenvolvimento de um sistema Web de Apoio ao Gerenciamento de Configuração dos Laboratórios de Informática do IFSul Câmpus Charqueadas, para controle de inventario dos mesmos. Baseado em um estudo do framework ITIL, no que se refere ao Gerenciamento de Configuração afim de implementar o OCS que era até então a ferramenta que mais se alinha ao contexto do setor de TI do IFSul Câmpus Charqueadas, configurando uma estação gerente/servidor, compreendendo o uso dos agentes do OCS e a sua base dados e, por fim, 0 desenvolvimento do módulo Web.

A partir dos estudos realizados e do protótipo implementado, entende-se que foi possível alcançar o objetivo do trabalho, uma vez que tem-se um protótipo funcional que poderá servir como base para a implantação de rotinas automatizadas de gestão da configuração no IFSul Câmpus Charqueadas.

\section{REFERÊNCIAS}

[1] U. Piazza, "O impacto da tecnologia da informação nas organizações," 2008, disponível em: $\quad$ ihttps://uilsonpiazza.wordpress.com/2008/09/17/oimpacto-da-tecnologia-dainformacao-nas-organizacoes/. Acessado em: Julho 2018.

[2] G. Consulting, "Por que investir em um suporte 247 para o setor de ti?" 2017, disponível em: ihttps://gaea.com.br/por-que-investir-em-umsuporte-24x7-para-o-setor-deti/. Acessado em: Julho 2018.

[3] M. A. D. S. Freitas, Fundamentos em Gerenciamento de TI, 2nd ed. Rio de Janeiro, RJ, BRA: Cabinet Office, 2013.

[4] A. Jacobuti, "O que e gerenciamento de serviços de ti?" 2016, disponível em: ihttp://www.itsmnapratica.com.br/o-que-e-gerenciamentode-servicos-de-ti/. Acesso em: Junho 2018.

[5] P. R. Foina, Tecnologia de Informação Planejamento de Gestão, 2nd ed. São Paulo, SP, BRA: Editora Atlas S.A, 2009.

[6] I. L. Magalhães and W. B. Pinheiro, Gerenciamento de Serviços de TI na Prática ， 1st ed. Sao Paulo, SP, BRA: Novatec Editora LTDA, 2007.

[7] Techoje, "Empresas aderem a modelos de gestão de ti," 2007, disponível em: "http://www.techoje.com.br/site/techoje/categoria/detalhe artigo/456"/. Acessado em: Julho 2018.

[8] P. Educação, "BDGC: Informações para melhores prática da itil," 2013, disponível em: https://www.portaleducacao.com.br/conteudo/artigos/direito/bdgcinformacoes-paramelhores-praticas-da-itil/46749/. Acessado em: Julho 2018.

[9] S. D. Castilho, D. A. de Souza Moraes, F. L. Velloso, and W. Oliveira, "Sistemas para documentação de ativos de tecnologias da informação," Caderno de Estudos Tecnológicos', vol. 1, no. 1, 2013.

[10] A. O. de Castro Ferreira, "Controlo e monitorização do parque informático," 2011. 
[11] E. GD, "As 4 melhores ferramentas para monitoramento de rede," 2016, disponível em: https://gdsolutions.com.br/infraestrutura/as-4-melhoresferramentas-para-monitoramentode-rede/. Acesso em: Julho 2018.

[12] A. TI, "7 ferramentas de monitoramento de rede essenciais para técnicos," 2017, disponível em: https://arquivoti.net/7-ferramentasde-monitoramento-de-rede-essenciaispara-tecnicos/. Acesso em: Julho 2018.

[13] L. C. de Mello and A. B. dos Santos, "Inventario automatizado de' equipamentos em redes de computadores," 2011

[14] OCS, "Ocs inventory professionnel." [Online]. Available: https: //ocsinventory-ng.org/?lang=en

[15] J. Alves, "Inventário de rede com ocs inventory," 2017, disponível em: ihttp://blog.4linux.com.br/inventario-de-rede-com-ocs-inventory/. Acesso em: Julho 2018.

[16] D. Scota, "Ocs inventory - instalação e configuração," 2018, disponível em: ihttps://daniel.scota.com.br/?p=717/. Acesso em: Julho-2018.

[17] S. F. Mattar, "Implantação de sistema de inventário e service desk com o ocs inventory e o glpi em uma empresa de advocacia," p. 86, 2012.

[18] OCS, "Ocs inventory github professionnel." [Online]. Available: https://github.com/OCSInventory-NG

[19] V. Linux, "Tutorial instalação gerente ocs." [Online]. Available: https://www.vivaolinux.com.br/artigo/ OCS-NG-Inventory-no-Debian-8-Jessie-Instalacao-eConfiguracao? página $=2$

[20] S. J. F. Lopes, "Gerenciamento de serviços de ti em instituição de ensino superior," p. 15, 2015.

[21] N. S. Honda, "Gaia inventário: Um modelo para gestão da configuração, inventário e ativos de serviços de tecnologia da informação." p. 51, 2016. [22] GLPI, "Glpi project." [Online]. Available: https://glpi-project.org/pt-br/

[22] Zabbix, "Zabbix enterprise-ready." [Online]. Available: https://www.zabbix.com/ 